\title{
Age-related differences in the cloacal microbiota of a wild bird species
}

\author{
Wouter FD van Dongen ${ }^{1 *}$, Joël White ${ }^{1,2}$, Hanja B Brandl', Yoshan Moodley ${ }^{1}$, Thomas Merkling ${ }^{2}$, Sarah Leclaire², \\ Pierrick Blanchard ${ }^{2}$, Étienne Danchin ${ }^{2}$, Scott A Hatch ${ }^{3}$ and Richard H Wagner ${ }^{1}$
}

\begin{abstract}
Background: Gastrointestinal bacteria play a central role in the health of animals. The bacteria that individuals acquire as they age may therefore have profound consequences for their future fitness. However, changes in microbial community structure with host age remain poorly understood. We characterised the cloacal bacteria assemblages of chicks and adults in a natural population of black-legged kittiwakes (Rissa tridactyla), using molecular methods.
\end{abstract}

Results: We show that the kittiwake cloaca hosts a diverse assemblage of bacteria. A greater number of total bacterial OTUs (operational taxonomic units) were identified in chicks than adults, and chicks appeared to host a greater number of OTUs that were only isolated from single individuals. In contrast, the number of bacteria identified per individual was higher in adults than chicks, while older chicks hosted more OTUs than younger chicks. Finally, chicks and adults shared only seven OTUs, resulting in pronounced differences in microbial assemblages. This result is surprising given that adults regurgitate food to chicks and share the same nesting environment.

Conclusions: Our findings suggest that chick gastrointestinal tracts are colonised by many transient species and that bacterial assemblages gradually transition to a more stable adult state. Phenotypic differences between chicks and adults may lead to these strong differences in bacterial communities. These data provide the framework for future studies targeting the causes and consequences of variation in bacterial assemblages in wild birds.

Keywords: Age-differences, Automated ribosomal intergenic spacer analysis, Bacteria, Black-legged kittiwakes, Cloaca, Gastrointestinal tract

\section{Background}

In animals, bacterial communities occur both externally (e.g. skin, feather, scales) and internally (e.g. gastrointestinal and reproductive tracts) of their hosts [1]. Although many gastrointestinal bacteria are host commensal, others are adaptive by being central in fundamental physiological processes [2-4], or have pathogenic effects [5]. Given the potentially profound impact of the gastrointestinal microbiota on host condition, the study of bacterial assemblages within animals is of high ecological and evolutionary importance [6].

\footnotetext{
* Correspondence: wouter.v.dongen@gmail.com

'Konrad Lorenz Institute of Ethology, Department of Integrative Biology and Evolution, University of Veterinary Medicine Vienna, Savoyenstrasse 1a, 1160 Vienna, Austria

Full list of author information is available at the end of the article
}

One of the central questions in the study of microbial communities in animals is how they are acquired and how the structure of communities varies with age. Young animals either passively or actively acquire microbiota from the environment after birth or hatching [6-8]. The gaxstrointestinal tract of young individuals is typically characterised by a high number of transient microbial species before eventually transitioning to a relatively stable state as adults [e.g. 9]. However, it remains unclear how microbial communities change throughout the lifetime of their hosts and how these fluctuations affect host health, behaviour and fitness [6]. Temporal variation in microbial composition may have important consequences for factors such as nutrition e.g. [8,10], mating preferences e.g. [11] and susceptibility to diseases e.g. [12]. It is therefore necessary to characterise bacterial communities at different life history stages of animal hosts. Scant information exists,

\section{Biomed Central}

(c) 2013 van Dongen et al.; licensee BioMed Central Ltd. This is an Open Access article distributed under the terms of the Creative Commons Attribution License (http://creativecommons.org/licenses/by/2.0), which permits unrestricted use, distribution, and reproduction in any medium, provided the original work is properly cited. 
however, and much past research has focussed on humans or other mammal species $[7,8]$.

Birds comprise a useful model for studying the causes and consequences of variation in bacterial assemblages. For example, the contrasting life histories of birds and mammals may result in differences in the mechanisms of microbial colonisation. Mammals, for example, acquire important maternal microbes during the birth process [9], whereas birds are more likely to acquire microbes after hatching from other sources, such as the nesting environment or food [7]. Second, many bird species regurgitate food to their young, thus permitting a mode of vertical microbial transmission that most mammals lack. Last, birds possess a cloaca, which serves the dual functions of excretion and gamete transfer. Gastrointestinal bacteria may thus be transferred during copulation [13], providing the potential for pathogens $[14,15]$ or beneficial strains to be sexually transmitted [16].

The few avian studies on the effect of age on gastrointestinal assemblages have mostly been conducted on domestic birds e.g. [17-20]. However, the artificial rearing conditions in which captive animals are raised make it difficult to extrapolate knowledge of bacterial assemblages in captive animals to their wild counterparts $[21,22]$. Moreover, the few studies in wild birds typically examine only a few target bacterial species and do not use community-level approaches to test for host age differences in bacterial assemblages e.g. [23-26], but see [10]. Very little is therefore known about how the normal gastrointestinal bacterial communities change between young and adult wild birds. This information forms an important basis for understanding bacterial acquisition and transmission mechanisms, the prevalence of enteropathogens and the consequences of particular communities of bacteria for host health.

Here, we characterise the bacterial assemblages of both adults and chicks in a wild population of black-legged kittiwakes (Rissa tridactyla), a pelagic gull with a wide distribution. We focus our analyses on cloacal assemblages because they are known to approximate assemblages deeper within the gastrointestinal tract [27] while requiring relatively non-invasive sampling methods. We have previously used a molecular technique [ARISA - Automated Ribosomal Intergenic Spacer Analysis: [28] to explore variation in cloacal bacteria assemblages in kittiwakes [13]. Although ARISA allows the rapid assessment of bacterial assemblage diversity, it does not permit species identification. We have therefore further developed a comprehensive ribosomal RNA (rRNA) clone library to identify actual bacterial species. Combined with ARISA, this allows the rapid assessment of actual species in bacterial assemblages, thus providing a framework for studying the causes and consequences of microbial assemblage variation in kittiwakes. We then use a community-based approach to test whether age predicts bacterial assemblage composition in kittiwakes and which bacterial lineages differ between chicks and adults.

\section{Methods}

Cloacal bacterial assemblages were sampled from 22 adult and 21 black-legged kittiwake chicks captured at nests in an abandoned U.S. Air Force radar tower on Middleton Island $\left(59^{\circ} 26^{\prime} \mathrm{N}, 146^{\circ} 20^{\prime} \mathrm{W}\right)$ in the Gulf of Alaska [13]. As an aim of our study was to taxonomically classify ARISA peaks (see below), it was necessary to obtain a comprehensive sample of the most common bacterial species present in chicks and adults. We therefore only sampled unrelated individuals (to minimise the overlap in bacterial assemblages between two individuals due to, for example, a shared nesting environment). In addition, chicks of a wide range of ages were sampled (range $=5-30$ days, mean $=$ 16.0 \pm 8.3 SD days) as bacterial assemblages were hypothesised to change rapidly with age. Sampling occurred between May and July 2009. Cloacal sampling and DNA extraction protocols are outlined in detail in White et al. 2010 [13]. We also regularly collected control samples during field work by pipetting $1 \mathrm{ml}$ of saline solution into a sterile vial from the saline stock, which ensured that all bacteria identified were of cloacal origin and not due to contamination of the stock solution or of reagents used for DNA extraction.

\section{Automated ribosomal intergenic spacer analysis}

We first characterised cloacal bacterial assemblages by performing PCR-ARISA, which exploits the extreme interspecies variability in the length of the intergenic spacer (IGS) lying between the conserved $16 \mathrm{~S}$ and $23 \mathrm{~S}$ rRNA genes in the bacterial ribosomal operon. ARISA involves the amplification of DNA extracted from the bacterial assemblage of interest using a fluorescentlylabelled primer and subsequent high-resolution electrophoresis in an automated system. Assemblages are therefore characterised by a series of electrophoretic peaks that vary according to the length of the amplified IGS fragment of each bacterial species. Given that unrelated species may share the same IGS fragment length, the number of species represented by each ARISA peak remains uncertain. This method has been optimised for the characterisation of cloacal bacterial assemblages in kittiwakes [13]. Briefly, the 16S-23S rRNA intergenic spacer was amplified using the FAM (6-carboxyfluorescein)-labeled primer S-D-Bact-1522-b-S-20 (5'-[6FAM] TGCG GCTGGATCCCCTCCTT-3') and the unlabelled LD-Bact-132-a- A-18 (5'-CCGGGTTTCCCCATTCGG-3'). In addition to amplifying the highly variable IGS region, the primers also amplify a $131 \mathrm{bp}$ fragment of the $23 \mathrm{~S}$ rRNA gene, which we used for subsequent phylogenetic analyses (see below). 
Fragment amplification was performed using PCR in $20 \mu \mathrm{l}$ reactions containing $0.2 \mathrm{mM}$ dNTPs, $1.5 \mathrm{mM} \mathrm{MgCl}_{2}$, $0.25 \mu \mathrm{M}$ of each primer, and 1 unit of GoTaq DNA polymerase with $1 \times$ buffer (Promega). Due to the coextraction of host and environmental DNA, it was not possible to accurately measure the concentration of the extracted DNA. Therefore, a standard of $4 \mu \mathrm{l}$ of eluted DNA extract was used per reaction. PCR was conducted using the following program: $94^{\circ} \mathrm{C}$ for 10 minutes, followed by 30 cycles of $94^{\circ} \mathrm{C}$ for 1 minute, $55^{\circ} \mathrm{C}$ for 1 minute and $72^{\circ} \mathrm{C}$ for $1 \mathrm{mi}$ nute, and a final elongation step at $72^{\circ} \mathrm{C}$ for 10 minutes. The successful amplification of the samples was confirmed on a $2 \%$ agarose gel and all PCR products were then diluted by a ratio of 1 in 30 . One microlitre of the diluted product was then added to $8.7 \mu$ l Hidi Formamide (Applied Biosystems) and $0.3 \mu \mathrm{l}$ of in-house designed ROX size standard (size standard range $=105-1007 \mathrm{bp}$ ). The mixture was then run on an ABI PRISM $3130 \times 1$ automated sequencer (Applied Biosystems). Traces were viewed on GeneMapper software 4.0 (Applied Biosystems).

\section{IGS-23S rRNA library construction and analysis}

We created an IGS-23S rRNA clone library of our samples in order to taxonomically classify ARISA peaks. We defined three hierarchical levels of units in our analyses: ARISA peaks, clones and OTUs (operational taxonomic units). As stated above, an ARISA peak is defined as one electrophoretic peak in an ARISA profile. Cloned fragments in our IGS-23S rRNA library were referred to as clones. During each cloning event we typically isolated multiple copies of the same sequence. Each unique clone (i.e. those with a unique sequence) was assumed to correspond to one unique ARISA peak. Finally, when multiple unique clones shared the same $23 \mathrm{~S}$ rRNA sequence and differed from each other only within the IGS, we assumed that they belonged to the same OTU. This approach in OTU classification is conservative but justified given that many bacterial species have multiple operons in their genomes that result in multiple ARISA peaks per OTU [29].

In developing our rRNA library, IGS-23S fragments of our bacterial samples were amplified as outlined above, except that both primers were unlabelled. Amplified products were then cloned using a TOPO TA cloning kit (Invitrogen) as per the manufacturer's protocol. We then picked between 8 and 96 transformed colonies for each cloacal sample and amplified the inserts using colony PCR in $25 \mu \mathrm{l}$ reactions containing $2.5 \mathrm{mM} \mathrm{MgCl}_{2}, 0.1 \mathrm{mM}$ dNTPs, $0.2 \mu \mathrm{M}$ M13 forward and reverse primer, and 2.5 units of Firepol polymerase and $1 \times$ buffer (Solis BioDyne). Reactions were run at $94^{\circ} \mathrm{C}$ for 5 minutes, followed by 35 cycles of $94^{\circ} \mathrm{C}$ for $30 \mathrm{~s}, 55^{\circ} \mathrm{C}$ for $30 \mathrm{~s}$ and $72^{\circ} \mathrm{C}$ for $30 \mathrm{~s}$, and a final extension at $72^{\circ} \mathrm{C}$ for $10 \mathrm{~min}$. The successful amplification of the samples was confirmed on a $2 \%$ agarose gel and primers and excess dNTPs were removed from the amplified products by digestion with exonucleaseshrimp and alkaline phosphatase (Fermentas Life Sciences). Cloned PCR products were amplified in both directions using Big Dye chemistry (Applied Biosystems) and sequenced on an ABI PRISM 3130×l automated sequencer (Applied Biosystems). Sequence editing and alignment was conducted using CLC DNA Workbench 6.1 (CLC bio). Rarefaction analyses were conducted using Analytic Rarefaction 1.3 (available at http://strata.uga.edu/software) to ascertain the comprehensiveness of our cloning effort.

\section{Comparison of ARISA peak size and clone fragment lengths}

One aim of our study was to identify the bacterial OTUs present in each cloacal sample of chicks and adults based entirely on the ARISA output. We therefore compared the length of cloned sequences with the peaks present in the ARISA profiles. We assigned each clone for each individual to an ARISA peak, taking into account the presence or absence of the ARISA peak and clone sequence in the other cloned samples for that age class. Any peak to which we did not assign a sequence was assumed to be an artefact and was therefore eliminated from subsequent analyses.

\section{Bacterial taxonomy}

A Basic Local Alignment Search Tool search BLAST: [30] was implemented on the $23 \mathrm{~S}$ rRNA sequence of cloned fragments to screen for published sequences that closely match our cloned sequences. The maximum BLAST score (typically > 200) and Expect-value (typically $<1 \times 10^{-50}$ ) were used as indicators of the strength of sequence similarity.

As BLAST provides information on similarity between two sequences but not on relatedness among sequences, we employed phylogenetic analyses to infer the taxonomic classification of the clones. We included all unique clones as well as two to four similar sequences for each clone from known species identified by BLAST. Both maximum-likelihood and Bayesian methods were used to ascertain the phylogenetic position of each unidentified species relative to known bacterial species. Given the immense diversity among bacteria, we conducted analyses separately for each phylum identified (Firmicutes, Actinobacteria and Proteobacteria). It was necessary to further separate the Proteobacteria into classes (Alphaproteobacteria, Betaproteobacteria and Gammaproteobacteria). Maximum-likelihood trees were constructed in Treefinder [31] using the nucleotide substitution model suggested by the Bayesian information criterion. Bootstrap support was estimated after 1000 replicates. Bayesian phylogenies were constructed in MrBayes 3.1.2 [32] using the same substitution model as suggested by Treefinder. Posterior probabilities were 
estimated after 10 million generations, sampling every 1000 generations.

The most derived phylogenetic position for each clone was then estimated based on a combination of the BLAST result and the phylogenetic trees. For example, when both BLAST and the phylogeny agreed that a particular clone belonged to a specific genus, we classified the clone to that genus. However, if there was disagreement between BLAST and the phylogenetic tree in the phylogenetic position of the clone, we classified the clone to a higher taxonomic level (e.g. class or family), with an emphasis on the position of the clone within the tree. Full sequences for each OTU have been deposited in GenBank under Accession numbers KC462595 - KC462734.

\section{Bacterial assemblage comparisons}

To characterise differences in the bacterial assemblages between chicks and adults, we implemented Unifrac [33] to compare overall bacterial assemblage composition using phylogenetic information. All assemblage analyses were conducted on the ARISA data and without abundance weights. Using the cloning data may produce misleading results due to stochastic variation in the identity of sequences cloned for each individual.

A $23 \mathrm{~S}$ rRNA phylogenetic tree containing all sequences found in chicks and adults was used for the analyses. We then implemented Unifrac to test 1) whether bacterial assemblages cluster by age (i.e. adults and chicks) using both the environmental clustering analysis (setting 'number of sequences to keep' to five and 'number of permutations' to 100 ) and principal coordinates analysis, 2) whether any clustering is statistically significant using both the P test (which does not use phylogenetic information; 100 permutations) and the Unifrac test (which tests whether more evolutionary history of bacterial assemblages is shared within age classes than between classes; 100 permutations) and 3) which bacterial lineages are responsible for differences in assemblages between the age classes, via the lineage-specific analysis (setting 'minimum descendants' to four).

A greater quantity of bacterial DNA could be extracted from adults than chicks (data not shown), probably due to the larger volume of saline solution that could be used to flush adult cloacae. Cloning of bacterial DNA fragments therefore tended to be more successful for adults than chicks and twice as many clones were sequenced per individual for adults compared to chicks (number of clones sequenced: adults $-47.0 \pm 28.0$ clones, chicks $-20.2 \pm 6.7$ clones, Mann Whitney $\mathrm{U}=434.0, \mathrm{P}<0.001)$. This agerelated discrepancy could lead to biases in downstream analyses in, for example, our estimates of age differences in the number of species hosted per individual. This, however, does not appear to be the case. First, the proportion of ARISA peaks that were discarded because they could not be assigned OTUs did not differ between adults and chicks (proportion of peaks discarded per individual: adults $-0.63 \pm 0.13$, chicks $-0.66 \pm 0.14, \mathrm{~F}_{1,41}=0.534$, $\mathrm{P}=0.469$ ), although the absolute number discarded was higher in adults (number of peaks discarded per individual: adults $-12.7 \pm 5.9$ peaks, chicks $-7.6 \pm 1.6$ peaks, Mann Whitney $\mathrm{U}=101.0, \mathrm{P}=0.001$ ) probably because higher quantities of bacterial DNA in adults produced more noise in the ARISA profiles. Second, cloning effort did not differ between chicks of different ages, both when correlating chick age with number of clones sequenced per individual $\left(\mathrm{r}=0.273, \mathrm{~F}_{1,19}=1.528, \mathrm{P}=0.231\right)$ or in a univariate test grouping chicks into young (5-10 days old) and old individuals (20-30 days old; number of clones sequenced per individual - young chicks: $18.5 \pm 4.5$ clones, old chicks: $22.4 \pm 8.3$ clones, $\left.\mathrm{F}_{1,19}=1.966, \mathrm{P}=0.177\right)$. Last, our Unifrac analyses are unaffected by these potential biases as we kept a minimum of five sequences for the clustering analyses and ran 100 permutations on the data. All nongenetic statistical analyses were conducting using SPSS 17.0 (SPSS, Chicago, Illinois, USA). All data are presented as mean \pm standard deviation.

\section{Results \\ Comparison of ARISA peak size and clone fragment lengths}

A mean of $33.9 \pm 24.4$ clones per individual were sequenced (range $=8-120$ clones per individual). Clones ranged in size from 304 bp to 1090 bp (Table 1). In total, we identified 142 unique clones, of which 99 were successfully matched to ARISA peaks. In the remaining 43 cases, we were unable to match peaks to clones because the clone was rare, resulting in low confidence of assignment. Due to the relatively high proportion of OTUs for which we could not assign ARISA peaks, the analyses presented below (e.g. differences in bacterial assemblages between adults and chicks) apply only to the most common OTUs found in chicks and adults.

The 142 unique clones corresponded to 76 OTUs. This discrepancy was due to indels causing variation in clone lengths for several OTUs. For example, although some OTUs were always consistent in clone length (e.g. OTU 2, Table 1), others were more variable (e.g. OTU 5 varied between 342 and $345 \mathrm{bp}$ in length). In most cases, one ARISA peak corresponded to a single OTU. However, in 16 cases, one ARISA peak was assigned to two distantlyrelated OTUs. For example, the 348 bp peak corresponded to species within the genera Corynebacterium and Athromitus, while the 544 bp peak corresponded to species of Rothia and Staphylococcus. The mean number of ARISA peaks per OTU was $1.5 \pm 1.5$ (range $1-9$ ). Of the 76 OTUs identified, 64 were of probable cloacal origin. The remaining 12 were also isolated from control samples and so were assumed to be contaminants (Additional file 1). 
Table 1 Identity of OTUs isolated from cloacae of black-legged kittiwakes

\begin{tabular}{|c|c|c|c|c|c|c|c|c|}
\hline OTU & $\begin{array}{l}\text { ARISA } \\
\text { peak }\end{array}$ & Clone & Difference & BLAST match & $\begin{array}{l}\text { BLAST } \\
\text { score }\end{array}$ & $\begin{array}{l}\text { Most derived } \\
\text { phylogenetic position }\end{array}$ & Phylum & Found in \\
\hline 1 & 304 & 308 & +4 & Peptoniphilus asaccharolyticus & 152 & Genus: Peptoniphilus & Firmicutes & Ad \\
\hline 1 & 305 & 309 & +4 & Peptoniphilus asaccharolyticus & 152 & Genus: Peptoniphilus & Firmicutes & $\mathrm{Ad}$ \\
\hline 1 & 396 & 399 & +3 & Peptoniphilus asaccharolyticus & 152 & Genus: Peptoniphilus & Firmicutes & Ad \\
\hline 1 & 397 & 400 & +3 & Peptoniphilus asaccharolyticus & 152 & Genus: Peptoniphilus & Firmicutes & $\mathrm{Ad}$ \\
\hline 1 & 398 & 401 & +3 & Peptoniphilus asaccharolyticus & 152 & Genus: Peptoniphilus & Firmicutes & $\mathrm{Ad}$ \\
\hline 2 & 313 & 315 & +2 & Enterococcus pseudoavium & 228 & Genus: Enterococcus & Firmicutes & $\mathrm{Ch}$ \\
\hline 3 & 331 & 331 & 0 & Enterococcus faecalis & 211 & Order: Lactobacillales & Firmicutes & $\mathrm{Ad}, \mathrm{Ch}$ \\
\hline 3 & 432 & 433 & +1 & Enterococcus faecalis & 211 & Order: Lactobacillales & Firmicutes & $\mathrm{Ad}, \mathrm{Ch}$ \\
\hline 4 & 340 & 341 & +1 & Enterococcus malodoratus & 239 & Genus: Enterococcus & Firmicutes & $\mathrm{Ch}$ \\
\hline 4 & 341 & 342 & +1 & Enterococcus malodoratus & 239 & Genus: Enterococcus & Firmicutes & Ch \\
\hline 4 & 433 & 434 & +1 & Enterococcus malodoratus & 239 & Genus: Enterococcus & Firmicutes & $\mathrm{Ch}$ \\
\hline 5 & 345 & 342 & -3 & Corynebacterium propinquum & 239 & Genus: Corynebacterium & Actinobacteria & $\mathrm{Ad}$ \\
\hline 5 & 346 & 343 & -3 & Corynebacterium propinquum & 239 & Genus: Corynebacterium & Actinobacteria & $\mathrm{Ad}$ \\
\hline 5 & 347 & 344 & -3 & Corynebacterium propinquum & 239 & Genus: Corynebacterium & Actinobacteria & $\mathrm{Ad}$ \\
\hline 5 & 348 & 345 & -3 & Corynebacterium propinquum & 239 & Genus: Corynebacterium & Actinobacteria & $\mathrm{Ad}$ \\
\hline 6 & & 342 & & Enterococcus malodoratus & 206 & Order: Lactobacillales & Firmicutes & Ch \\
\hline 7 & 348 & 349 & +1 & Arthromitus sp. & 233 & Genus: Arthromitus & Firmicutes & $\mathrm{Ad}, \mathrm{Ch}$ \\
\hline 7 & 418 & 419 & +1 & Arthromitus sp. & 233 & Genus: Arthromitus & Firmicutes & $\mathrm{Ad}, \mathrm{Ch}$ \\
\hline 8 & & 349 & & Lactobacillus salivarius & 206 & Genus: Lactobacillus & Firmicutes & $\mathrm{Ch}$ \\
\hline 9 & 349 & 350 & +1 & Lactobacillus animalis & 213 & Genus: Lactobacillus & Firmicutes & $\mathrm{Ad}, \mathrm{Ch}$ \\
\hline 9 & 440 & 443 & +3 & Lactobacillus animalis & 213 & Genus: Lactobacillus & Firmicutes & $\mathrm{Ad}, \mathrm{Ch}$ \\
\hline 9 & 441 & 444 & +3 & Lactobacillus animalis & 213 & Genus: Lactobacillus & Firmicutes & $\mathrm{Ad}, \mathrm{Ch}$ \\
\hline 9 & 519 & 520 & +1 & Lactobacillus animalis & 213 & Genus: Lactobacillus & Firmicutes & $\mathrm{Ad}, \mathrm{Ch}$ \\
\hline 9 & 520 & 521 & +1 & Lactobacillus animalis & 213 & Genus: Lactobacillus & Firmicutes & $\mathrm{Ad}, \mathrm{Ch}$ \\
\hline 10 & 352 & 353 & +1 & Aerococcus urinae & 222 & Order: Lactobacillales & Firmicutes & $\mathrm{Ad}$ \\
\hline 11 & 357 & 354 & -3 & Corynebacterium aurimucosum & 196 & Genus: Corynebacterium & Actinobacteria & $\mathrm{Ad}$ \\
\hline 12 & & 357 & & Atopobium parvulum & 204 & Order: Clostridiales & Firmicutes & $\mathrm{Ad}$ \\
\hline 13 & & 357 & & Carnobacterium mobile & 237 & Genus: Carnobacterium & Firmicutes & $\mathrm{Ch}$ \\
\hline 13 & & 463 & & Carnobacterium mobile & 237 & Genus: Carnobacterium & Firmicutes & Ch \\
\hline 14 & & 357 & & Lactobacillus crispatus & 237 & Genus: Lactobacillus & Firmicutes & $\mathrm{Ad}$ \\
\hline 14 & 603 & 607 & +4 & Lactobacillus crispatus & 237 & Genus: Lactobacillus & Firmicutes & $\mathrm{Ad}$ \\
\hline 15 & & 361 & & Bacillus megaterium & 215 & Genus: Bacillus & Firmicutes & $\mathrm{Ch}$ \\
\hline 16 & 365 & 361 & -4 & Mycobacterium sp. & 161 & Suborder: Corynebacterineae & Actinobacteria & $\mathrm{Ad}$ \\
\hline 16 & 366 & 362 & -4 & Mycobacterium sp. & 161 & Suborder: Corynebacterineae & Actinobacteria & $\mathrm{Ad}$ \\
\hline 16 & 367 & 363 & -4 & Mycobacterium sp. & 161 & Suborder: Corynebacterineae & Actinobacteria & $\mathrm{Ad}$ \\
\hline 16 & 368 & 364 & -4 & Mycobacterium sp. & 161 & Suborder: Corynebacterineae & Actinobacteria & Ad \\
\hline 16 & 369 & 365 & -4 & Mycobacterium sp. & 161 & Suborder: Corynebacterineae & Actinobacteria & $\mathrm{Ad}$ \\
\hline 16 & 371 & 367 & -4 & Mycobacterium sp. & 161 & Suborder: Corynebacterineae & Actinobacteria & $\mathrm{Ad}$ \\
\hline 17 & & 363 & & Oceanobacillus iheyensis & 187 & Class: Bacilli & Firmicutes & $\mathrm{Ch}$ \\
\hline 18 & 380 & 375 & -5 & Arcanobacterium abortisuis & 174 & Family: Actinomycetaceae & Actinobacteria & Ad \\
\hline 19 & & 377 & & Clostridium difficile & 217 & Genus: Clostridium & Firmicutes & $\mathrm{Ch}$ \\
\hline 20 & 376 & 378 & +2 & Enterococcus faecalis & 239 & Genus: Enterococcus & Firmicutes & $\mathrm{Ch}$ \\
\hline 20 & 478 & 480 & +2 & Enterococcus faecalis & 239 & Genus: Enterococcus & Firmicutes & $\mathrm{Ch}$ \\
\hline 21 & 392 & 388 & -4 & Corynebacterium aurimucosum & 182 & Genus: Corynebacterium & Actinobacteria & $\mathrm{Ad}$ \\
\hline
\end{tabular}


Table 1 Identity of OTUs isolated from cloacae of black-legged kittiwakes (Continued)

\begin{tabular}{|c|c|c|c|c|c|c|c|c|}
\hline 22 & 388 & 390 & +2 & Pediococcus inopinatus & 174 & Order: Lactobacillales & Firmicutes & $\mathrm{Ch}$ \\
\hline 23 & 392 & 395 & +3 & Clostridium piliforme & 219 & Genus: Clostridium & Firmicutes & $\mathrm{Ch}$ \\
\hline 23 & 393 & 396 & +3 & Clostridium piliforme & 219 & Genus: Clostridium & Firmicutes & $\mathrm{Ch}$ \\
\hline 24 & & 398 & & Acidimicrobineae sp. & 180 & Phylum: Actinobacteria & Actinobacteria & $\mathrm{Ad}$ \\
\hline 25 & & 400 & & Clostridium piliforme & 209 & Genus: Clostridium & Firmicutes & $\mathrm{Ad}$ \\
\hline 25 & 753 & 756 & +3 & Clostridium piliforme & 209 & Genus: Clostridium & Firmicutes & $\mathrm{Ad}$ \\
\hline 26 & 398 & 401 & +3 & Staphylococcus vitulinus & 243 & Genus: Staphylococcus & Firmicutes & $\mathrm{Ch}$ \\
\hline 26 & 399 & 402 & +3 & Staphylococcus vitulinus & 243 & Genus: Staphylococcus & Firmicutes & $\mathrm{Ch}$ \\
\hline 26 & 406 & 407 & +1 & Staphylococcus vitulinus & 243 & Genus: Staphylococcus & Firmicutes & $\mathrm{Ch}$ \\
\hline 26 & 430 & 431 & +1 & Staphylococcus vitulinus & 243 & Genus: Staphylococcus & Firmicutes & $\mathrm{Ch}$ \\
\hline 26 & 431 & 432 & +1 & Staphylococcus vitulinus & 243 & Genus: Staphylococcus & Firmicutes & $\mathrm{Ch}$ \\
\hline 26 & 489 & 492 & +2 & Staphylococcus vitulinus & 243 & Genus: Staphylococcus & Firmicutes & $\mathrm{Ch}$ \\
\hline 26 & 520 & 520 & 0 & Staphylococcus vitulinus & 243 & Genus: Staphylococcus & Firmicutes & Ch \\
\hline 26 & 521 & 521 & 0 & Staphylococcus vitulinus & 243 & Genus: Staphylococcus & Firmicutes & $\mathrm{Ch}$ \\
\hline 26 & & 621 & & Staphylococcus vitulinus & 243 & Genus: Staphylococcus & Firmicutes & $\mathrm{Ch}$ \\
\hline 27 & 421 & 422 & +1 & Bacillus sp. & 213 & Genus: Bacillus & Firmicutes & $\mathrm{Ch}$ \\
\hline 27 & 422 & 423 & +1 & Bacillus sp. & 213 & Genus: Bacillus & Firmicutes & $\mathrm{Ch}$ \\
\hline 27 & 570 & 568 & +2 & Bacillus sp. & 213 & Genus: Bacillus & Firmicutes & $\mathrm{Ch}$ \\
\hline 27 & 589 & 590 & +1 & Bacillus sp. & 213 & Genus: Bacillus & Firmicutes & $\mathrm{Ch}$ \\
\hline 28 & & 434 & & Streptococcus parauberis & 231 & Genus: Streptococcus & Firmicutes & $\mathrm{Ch}$ \\
\hline 29 & & 438 & & Acidimicrobiaceae sp. & 189 & Phylum: Actinobacteria & Actinobacteria & $\mathrm{Ch}$ \\
\hline 30 & 437 & 439 & +2 & Staphylococcus saprophyticus & 239 & Genus: Staphylococcus & Firmicutes & $\mathrm{Ch}$ \\
\hline 30 & 438 & 440 & +2 & Staphylococcus saprophyticus & 239 & Genus: Staphylococcus & Firmicutes & Ch \\
\hline 31 & 446 & 441 & -5 & Corynebacterium propinquum & 182 & Genus: Corynebacterium & Actinobacteria & $\mathrm{Ad}$ \\
\hline 32 & 443 & 445 & +3 & Bacillus globisporus & 206 & Genus: Bacillus & Firmicutes & $\mathrm{Ch}$ \\
\hline 33 & & 446 & & Bacillus cereus & 213 & Genus: Bacillus & Firmicutes & Ch \\
\hline 34 & 452 & 448 & -4 & Corynebacterium propinquum & 172 & Genus: Corynebacterium & Actinobacteria & $\mathrm{Ad}$ \\
\hline 34 & 458 & 454 & -4 & Corynebacterium propinquum & 172 & Genus: Corynebacterium & Actinobacteria & $\mathrm{Ad}$ \\
\hline 34 & 459 & 455 & -4 & Corynebacterium propinquum & 172 & Genus: Corynebacterium & Actinobacteria & $\mathrm{Ad}$ \\
\hline 34 & 460 & 456 & -4 & Corynebacterium propinquum & 172 & Genus: Corynebacterium & Actinobacteria & $\mathrm{Ad}$ \\
\hline 34 & 461 & 457 & -4 & Corynebacterium propinquum & 172 & Genus: Corynebacterium & Actinobacteria & $\mathrm{Ad}$ \\
\hline 35 & 453 & 454 & +1 & Corynebacterium amycolatum & 200 & Genus: Corynebacterium & Actinobacteria & $\mathrm{Ad}$ \\
\hline 35 & 454 & 455 & +1 & Corynebacterium amycolatum & 200 & Genus: Corynebacterium & Actinobacteria & $\mathrm{Ad}$ \\
\hline 36 & 480 & 479 & -1 & Rhodococcus erythropolis & 207 & Genus: Rhodococcus & Actinobacteria & $\mathrm{Ad}$ \\
\hline 36 & 490 & 490 & 0 & Rhodococcus erythropolis & 207 & Genus: Rhodococcus & Actinobacteria & $\mathrm{Ad}$ \\
\hline 36 & 493 & 492 & -1 & Rhodococcus erythropolis & 207 & Genus: Rhodococcus & Actinobacteria & $\mathrm{Ad}$ \\
\hline 37 & 502 & 505 & +3 & Escherichia coli & 213 & Genus: Escherichia & Proteobacteria & $\mathrm{Ad}, \mathrm{Ch}$ \\
\hline 37 & 504 & 507 & +3 & Escherichia coli & 213 & Genus: Escherichia & Proteobacteria & $\mathrm{Ad}, \mathrm{Ch}$ \\
\hline 37 & 506 & 509 & +3 & Escherichia coli & 213 & Genus: Escherichia & Proteobacteria & $\mathrm{Ad}, \mathrm{Ch}$ \\
\hline 37 & 596 & 599 & +3 & Escherichia coli & 213 & Genus: Escherichia & Proteobacteria & $\mathrm{Ad}, \mathrm{Ch}$ \\
\hline 37 & 598 & 601 & +3 & Escherichia coli & 213 & Genus: Escherichia & Proteobacteria & $\mathrm{Ad}, \mathrm{Ch}$ \\
\hline 38 & & 508 & & Bacillus globisporus & 198 & Genus: Bacillus & Firmicutes & $\mathrm{Ch}$ \\
\hline 39 & & 509 & & Nocardia seriolae & 174 & Order: Actinomycetales & Actinobacteria & $\mathrm{Ch}$ \\
\hline 39 & & 514 & & Nocardia seriolae & 174 & Order: Actinomycetales & Actinobacteria & $\mathrm{Ch}$ \\
\hline 40 & 520 & 522 & +2 & Corynebacterineae sp. & 207 & Order: Actinomycetales & Actinobacteria & $\mathrm{Ad}$ \\
\hline
\end{tabular}


Table 1 Identity of OTUs isolated from cloacae of black-legged kittiwakes (Continued)

\begin{tabular}{|c|c|c|c|c|c|c|c|c|}
\hline 41 & 527 & 526 & -1 & Microbacterium testaceum & 198 & Genus: Microbacterium & Actinobacteria & $\mathrm{Ch}$ \\
\hline 42 & 542 & 536 & -2 & Rothia mucilaginosa & 174 & Genus: Rothia & Actinobacteria & $\mathrm{Ad}, \mathrm{Ch}$ \\
\hline 42 & 544 & 538 & -2 & Rothia mucilaginosa & 174 & Genus: Rothia & Actinobacteria & $\mathrm{Ad}, \mathrm{Ch}$ \\
\hline 43 & 544 & 545 & +1 & Staphylococcus aureus & 207 & Genus: Staphylococcus & Firmicutes & $\mathrm{Ad}$ \\
\hline 44 & & 546 & & Nocardia seriolae & 130 & Order: Actinomycetales & Actinobacteria & Ch \\
\hline 45 & & 554 & & Lactobacillus mucosae & 209 & Genus: Lactobacillus & Firmicutes & Ad \\
\hline 46 & 572 & 577 & +5 & Clostridium tyrobutyricum & 90 & Order: Clostridiales & Firmicutes & Ad \\
\hline 47 & & 580 & & Streptococcus pyogenes & 198 & Genus: Streptococcus & Firmicutes & Ad, Ch \\
\hline 47 & 707 & 712 & +5 & Streptococcus pyogenes & 198 & Genus: Streptococcus & Firmicutes & $\mathrm{Ad}, \mathrm{Ch}$ \\
\hline 47 & 713 & 718 & +5 & Streptococcus pyogenes & 198 & Genus: Streptococcus & Firmicutes & $\mathrm{Ad}, \mathrm{Ch}$ \\
\hline 47 & 716 & 721 & +5 & Streptococcus pyogenes & 198 & Genus: Streptococcus & Firmicutes & $\mathrm{Ad}, \mathrm{Ch}$ \\
\hline 47 & 785 & 788 & +3 & Streptococcus pyogenes & 198 & Genus: Streptococcus & Firmicutes & Ad, Ch \\
\hline 48 & 592 & 591 & -1 & Micrococcus luteus & 213 & Genus: Micrococcus & Actinobacteria & $\mathrm{Ch}$ \\
\hline 49 & 611 & 609 & -2 & Corynebacterium efficiens & 169 & Genus: Corynebacterium & Actinobacteria & $\mathrm{Ch}$ \\
\hline 50 & 611 & 614 & +3 & Streptococcus dysgalactiae & 213 & Genus: Streptococcus & Firmicutes & $\mathrm{Ad}$ \\
\hline 51 & & 615 & & Jonesia denitrificans & 182 & Suborder: Micrococcineae & Actinobacteria & $\mathrm{Ch}$ \\
\hline 51 & & 620 & & Jonesia denitrificans & 182 & Suborder: Micrococcineae & Actinobacteria & Ch \\
\hline 51 & & 621 & & Jonesia denitrificans & 182 & Suborder: Micrococcineae & Actinobacteria & $\mathrm{Ch}$ \\
\hline 51 & & 622 & & Jonesia denitrificans & 182 & Suborder: Micrococcineae & Actinobacteria & $\mathrm{Ch}$ \\
\hline 52 & 622 & 625 & +3 & Sinobacteraceae sp. & 211 & Order: Xanthomonadales & Proteobacteria & Ad \\
\hline 53 & & 626 & & Agrococcus sp. & 207 & Family: Microbacteriaceae & Actinobacteria & Ch \\
\hline 54 & & 634 & & Clavibacter michiganensis & 185 & Family: Microbacteriaceae & Actinobacteria & $\mathrm{Ch}$ \\
\hline 55 & & 670 & & Yersinia enterocolitica & 215 & Genus: Yersinia & Proteobacteria & Ad \\
\hline 56 & & 671 & & Herbaspirillum sp. & 217 & Genus: Herbaspirillum & Proteobacteria & $\mathrm{Ad}$ \\
\hline 57 & & 674 & & Arthrobacter arilaitensis & 198 & Family: Micrococcaceae & Actinobacteria & Ch \\
\hline 58 & & 692 & & Azoarcus sp. & 195 & Family: Rhodocyclaceae & Proteobacteria & $\mathrm{Ch}$ \\
\hline 59 & & 713 & & Agromyces sp. & 185 & Family: Microbacteriaceae & Actinobacteria & $\mathrm{Ch}$ \\
\hline 60 & & 723 & & Micrococcus luteus & 176 & Family: Micrococcaceae & Actinobacteria & Ch \\
\hline 61 & & 740 & & Arthrobacter arilaitensis & 182 & Family: Micrococcaceae & Actinobacteria & $\mathrm{Ch}$ \\
\hline 62 & 794 & 796 & +2 & Phenylobacterium zucineum & 165 & Family: Caulobacteraceae & Proteobacteria & Ad \\
\hline 63 & 973 & 974 & +1 & Bradyrhizobium elkanii & 209 & Genus: Bradyrhizobium & Proteobacteria & $\mathrm{Ad}, \mathrm{Ch}$ \\
\hline 63 & & 1047 & & Bradyrhizobium elkanii & 209 & Genus: Bradyrhizobium & Proteobacteria & Ad, Ch \\
\hline 63 & & 1048 & & Bradyrhizobium elkanii & 209 & Genus: Bradyrhizobium & Proteobacteria & $\mathrm{Ad}, \mathrm{Ch}$ \\
\hline 64 & & 1090 & & Bradyrhizobiaceae sp. & 198 & Genus: Bradyrhizobium & Proteobacteria & $\mathrm{Ch}$ \\
\hline
\end{tabular}

For each OTU, the following variables are displayed: OTU identification number, ARISA peak size, clone fragment length, the difference between the latter two variables (i.e. clone length minus ARISA peak size), the species identity of the most similar sequence in GenBank as determined by the BLAST algorithm, the maximum BLAST score, the most derived phylogenetic position of the OTU and the phylum to which the OTU belongs. In some cases, we could not determine which ARISA peak represented a particular clone (indicated by a blank space in the "ARISA peak" column).

A virtually perfect correlation existed between the size of ARISA peaks and clone length $\left(r=1.000, F_{1,95}=299,083\right.$, $\mathrm{P}<0.001$ ), although some discrepancies still existed (Table 1). Clone lengths differed from their corresponding ARISA peak sizes by a mean of $1.0 \pm 3.6 \mathrm{bp}$ (range -6 to $+15 \mathrm{bp}$ ).

\section{Bacterial taxonomy}

The resolution of OTU identification depended on both the strength of the BLAST match and the ability to resolve their respective phylogenetic positions (Additional file 2). The mean maximum BLAST score for each OTU was $199 \pm 25$ (range $=90-243$ ). In 64.5\% of cases we assigned an OTU to a specific genus, to a family in $14.5 \%$ of cases, to a suborder in $2.6 \%$ of cases, to an order in $13.2 \%$ of cases, to a class in $2.6 \%$ of cases and to a phylum in $2.6 \%$ of cases. The most common phylum represented by the cloacal assemblages was Firmicutes (50.0\%), followed by Actinobacteria $(37.5 \%)$ and Proteobacteria (12.5\% consisting of $4.7 \%$ 
Alphaproteobacteria, 3.1\% Betaproteobacteria and 4.7\% Gammaproteobacteria). The most common genera found were Corynebacterium (10.9\%) and Lactobacillus (6.6\%). The majority of OTUs identified in the control samples belonged to the class Alphaproteobacteria (58.3\%). In addition, we also identified bacteria from the classes Betaproteobacteria (16.7\%) and Gammaproteobacteria (8.3\%) and from the phylum Firmicutes (16.7\%).

\section{Bacterial assemblages in adults and chicks}

From our 23S rRNA cloning library, we identified 31 OTUs in adults and 40 OTUs in chicks, despite sequencing less than a quarter the number of chick to adult clones. Rarefaction analyses revealed that we had sampled the majority of common OTUs found in adults, but that many rare OTUs remained unidentified in chicks (Figure 1). Only seven OTUs were shared between the two age groups. Sixty three percent of OTUs identified in chicks were isolated from only single individuals while, in adults, $48 \%$ of OTUs were isolated from single individuals. From the ARISA data, chick age was positively correlated with number of OTUs hosted per individual $\left(r=0.452, \mathrm{~F}_{1,19}=4.889, \mathrm{P}=0.039\right.$; Figure 2$)$. In addition, adults hosted a higher number of OTUs per individual than chicks (mean number of OTUs per individual kittiwake: chicks $=5.8 \pm 3.0$ OTUs, adults $=9.2 \pm 2.3$ OTUs, $\mathrm{F}_{1,43}=17.556, \mathrm{P}<0.001$ ).

Analyses in Unifrac revealed strong clustering of the sequences by age (Figure 3). In addition, principal coordinates analysis separated chicks and adults along the first axis explaining $45.6 \%$ of the variation (PC1 scores chicks: $0.264 \pm 0.070$, adults: $-0.252 \pm 0.091, \mathrm{~F}_{1,41}=$ 430.5, $\mathrm{P}<0.001$; Figure 4), but not along the second axis, which explained $12.0 \%$ of variation, or the third

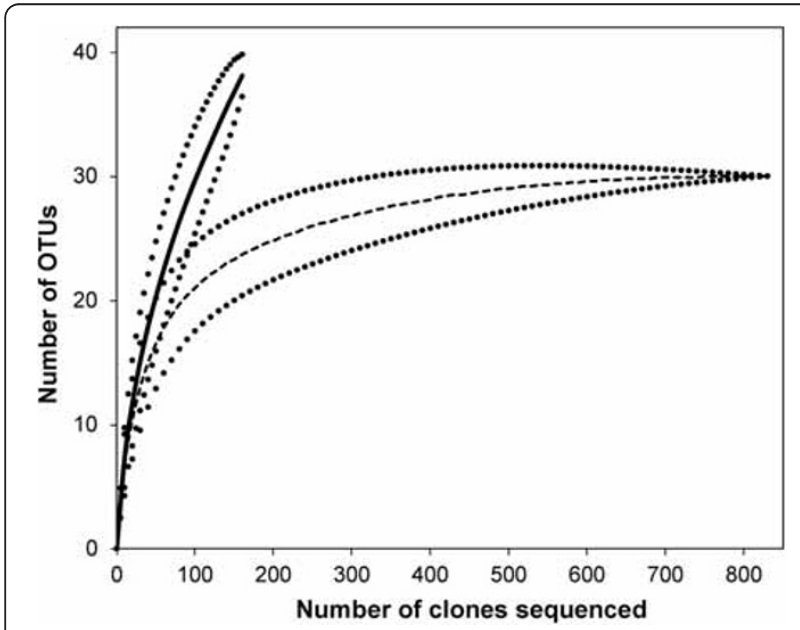

Figure 1 Rarefaction curves of OTU richness and cloning effort of cloacal bacteria of adult and chicks. Adults are represented by the dashed line and chicks by the solid line. Dotted lines represent 95\% confidence limits. axis, explaining $7.9 \%$ of variation (PC2 scores - chicks: $0.019 \pm 0.195$, adults: $0.018 \pm 0.099, \mathrm{Z}=-0.875, \mathrm{P}=$ 0.382 ; PC2 scores - chicks: $-0.019 \pm 0.195$, adults: $0.018 \pm 0.099, \mathrm{Z}=-0.462, \mathrm{P}=0.664)$.

The clustering by age was statistically significant ( $\mathrm{P}$ test in UniFrac: $\mathrm{P}=0.007$ ), although the clusters did not represent evolutionary divergence of bacteria between the age classes (Unifrac significance: $\mathrm{P}=0.104$ ). Lineage-specific analysis suggested that the age differences in bacterial assemblages were due to the presence of several OTUs in only one age class. For example, various OTUs of the genus Corynebacterium were abundant in adults (six OTUs identified, many of which were present in most individuals), but virtually absent in chicks (one OTU identified in only one individual; $\mathrm{P}<0.001$ ). Similarly, certain OTUs were only found in adults (e.g. OTU 1: Peptoniphilus sp., P < 0.001; OTU 18: Actinomycetaceae sp., $\mathrm{P}<0.001$; OTU 46: Clostridiales sp., $\mathrm{P}<0.001$ ), while others were unique to or more abundant in chicks (e.g. OTU 27 and 32; Bacillus sp., $\mathrm{P}<0.001$; OTU 37: Escherichia sp., $\mathrm{P}<0.001$ ).

\section{Discussion}

We identified 64 bacterial OTUs in the cloacae of adult and chick black-legged kittiwakes, a majority of which were identified as Firmicutes and Actinobacteria, while a smaller proportion belonged to the Proteobacteria. A greater number of total OTUs were identified in chick cloacae than in adults. However, the number of OTUs hosted per individual increased with age. Older chicks hosted more OTUs per individual than younger chicks and adults hosted more OTUs per individual than chicks. Surprisingly very little overlap existed in the bacterial assemblages between chicks and adults. Only seven of 64 OTUs were shared between the two age groups (e.g. OTU 3: order Lactobacillales, OTU 9: genus: Lactobacillus and OTU 37:

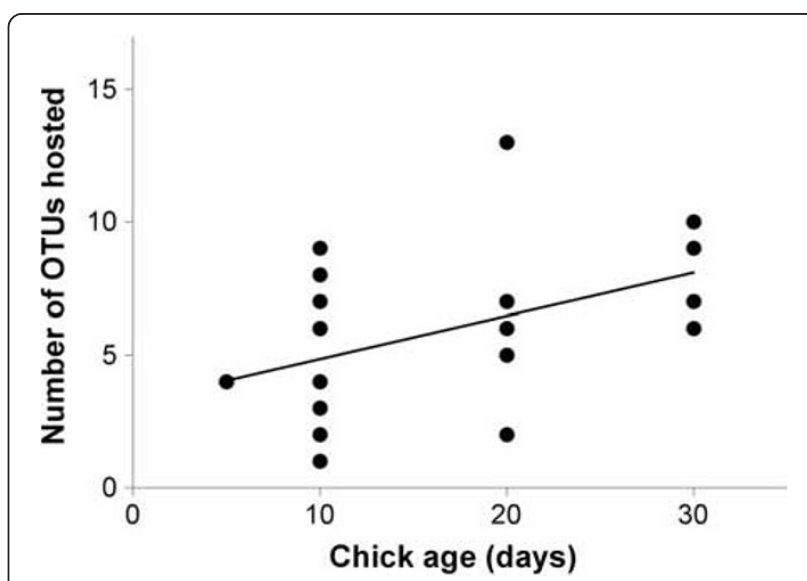

Figure 2 Age-related changes in the number of bacterial OTUs hosted by black-legged kittiwake chicks. 


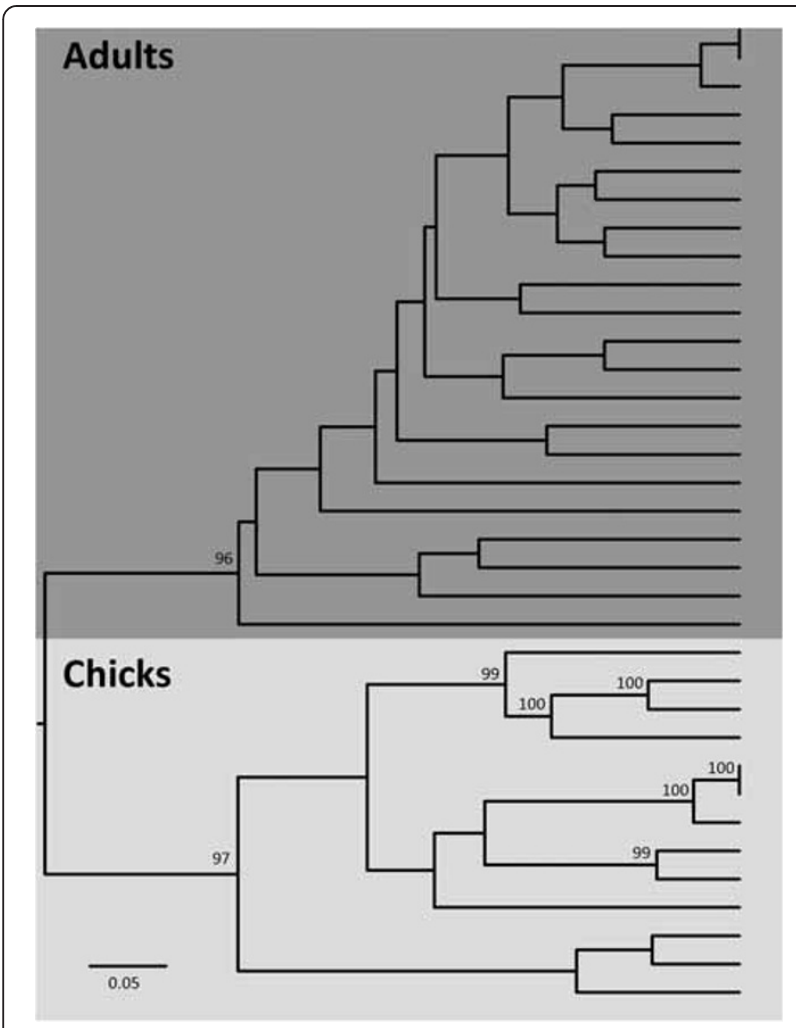

Figure 3 Unifrac clustering analysis of bacterial assemblages in the cloacae of adult and chick black-legged kittiwakes. Adults are represented by the dark grey box and chicks by the light grey box. Each leaf represents the bacterial assemblage of one individual kittiwake. Nodes with numbers were recovered more than $95 \%$ of the time during 100 permutations. Note that the analysis removed several chick individuals that harboured fewer than five OTUs (see Methods).

genus Escherichia), while the vast majority were found exclusively in only chicks or adults. For example, Corynebacterium was the most common genus of bacteria identified in adults, but it was virtually absent from chicks. This pronounced difference between adults and chicks resulted in strong statistical clustering of bacterial assemblages according to host age.

ARISA has been widely used to provide important insights in a wide range of fields within microbiology e.g. [34-38]. Coupled with a clone library for species identification, it represents a highly relevant research tool to allow the rapid and inexpensive characterisation of environmental bacterial assemblages. Despite the advantages of ARISA, this technique has some limitations (which are not all necessarily restricted to ARISA). For example, biases inherent during DNA extraction and PCR are also known to affect the apparent composition of bacterial assemblages $[39,40]$. ARISA can potentially underestimate species richness, as eight percent of bacterial species are known not to have the $23 \mathrm{~S}$ and $16 \mathrm{~S}$ rRNA organised in an operon (i.e. they have no intergenic spacer region) or have very large IGS lengths

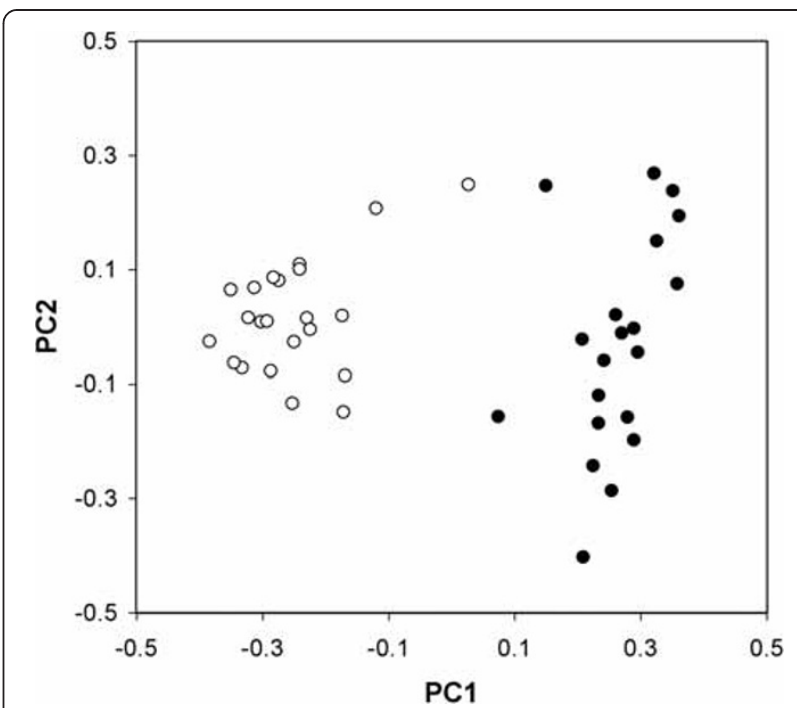

Figure 4 First two principal coordinates extracted from a principal coordinate analysis of kittiwake cloacal bacterial assemblages. Chicks are represented by closed circles and adults by open circles. The first principal component explained $45.6 \%$ of the variation, while the second explained $12.0 \%$.

that cannot be detected by ARISA [29]. In addition, divergent bacteria may share the same IGS length and therefore be associated with the same ARISA peak [this study, [29]. In contrast, some species have several operons in their genomes resulting in multiple ARISA peaks for single species and potentially leading to overestimates in diversity [29]. In our dataset, we conservatively assumed that any two clones with the same $23 \mathrm{~S}$ sequence derived from the same OTU. We consequently identified many OTUs with multiple operons (1-9 operons were identified per OTU). We also only included OTUs in our analyses for which we could confidently assign ARISA peaks, resulting in several genuine OTUs being excluded. This conservative approach means that our estimates of OTU richness are likely to represent a minimum. However, these biases apply equally for both chicks and adults and we were still able to identify a relatively large number of OTUs suitable for community-level comparisons.

The establishment of bacterial communities in the gastrointestinal tract of young animals is characterised by a high turnover of many transient species and large changes in community structure over short periods of time. For example, González-Braojos et al. 2012 [23] found that, in faecal sacs of nestling pied flycatchers (Ficedula hypoleuca), Enterobacteriaceae loads decreased when the nestlings aged from 7 to 13 days, while Enterococci loads concurrently increased. Age-related changes in the composition of other important gastrointestinal microbes, such as fungi, are also known to occur e.g. [25]. The rapid changes in bacterial community structure 
in young animals may arise due to a number of reasons including resource competition between bacterial species, shifts in host diet or age-related variation in the chemical and physiological state of the gastrointestinal tract $[8,10,23]$. For example, in humans, the early colonisation of the gut by facultative anaerobes (e.g. Enterococci and Enterobacteria) reduces gut oxygen levels which allows anaerobic bacteria to become established [8]. Eventually, gastrointestinal bacterial communities are known to transition to a stable adult state [7]. Adult bacterial communities may differ from those of young individuals due to the more developed immune system of adults [41-43], the low mobility of young animals resulting in a restricted environment from which to obtain bacteria or contrasting chemical and anatomical cloacal environments that are differentially hospitable or hostile to various bacteria $[7,8]$.

Our data support these findings. For example, the fact that the chick rarefaction curve failed to plateau and that more OTUs were unique to individual chicks than adults suggests that chicks host more rare, and presumably transient, bacterial species than adults. Second, our finding that adults host a greater number of OTUs per individual than chicks, and that the number of OTUs hosted by chicks increases with age, is in accordance with previous studies that have shown that species richness in bacterial assemblages increases as animals reach adulthood e.g. $[19,20,26]$. Last, we identified substantial variation in bacterial assemblage composition between chicks and adults, highlighting the dynamic nature of bacterial communities within the gut. It is, unfortunately, not possible to deduce the fitness consequences on hosts of agerelated changes in bacterial microbiota from our genetic data, especially given the great intrageneric diversity in ecological roles and pathogenicity of bacteria. It, for example, remains unknown why bacteria of the genus Corynebacterium are so prevalent in adults, but almost absent in chicks. However, some inferences can still be made. The seven OTUs shared between chicks and adults may be beneficial or commensal and therefore retained in the gastrointestinal microbiota as the hosts age. For example, two of the shared OTUs were Lactobacillales species, many of which are known to competitively exclude pathogenic bacteria and increase antibody levels, thus increasing immunity to pathogens $[44,45]$. Similarly, another shared OTU belonged to the genus Escherichia. Escherichia bacteria are common commensals in the gastrointestinal tract [46], which are known to rapidly colonise the gut of young birds $[18,19,24]$.

The data generated in this study will provide new opportunities to investigate the causes and consequences of variation in bacterial assemblages in a wild bird species. Although much is known in domesticated bird species, relatively little is known about the acquisition of gastrointestinal bacteria assemblages in wild birds. For example, assemblages are known to vary with both external factors, such as nutrition e.g. [47] and environment e.g. [48], and host-related traits such as genotype e.g. [49], body condition e.g. [48], immune system e.g. [50], and sex and mating behaviour e.g. [13]. Our data will allow us to build on these studies with large experimental data sets to explore how specific bacterial species are acquired (e.g. via cross-fostering experiments to ascertain how environment and genotype affect microbial assemblages in chicks) and how they impact on host condition and fitness (e.g. whether individuals that host different bacterial assemblages cf. enterotypes: [51] differ in condition or reproductive success).

\section{Conclusions}

The striking difference in bacterial assemblages between chicks and adults suggests that despite sharing the same nesting environment and being fed regurgitated food by their parents, others factors affect the acquisition of cloacal bacterial assemblages in kittiwakes. Although several previous studies in both domestic e.g. $[17,19,20]$ and wild birds e.g. $[10,24,25]$ have demonstrated agedifferences in gastrointestinal bacteria, we have done so at the community level rather than the single-species level. This approach has highlighted how strongly assemblages can differ between age-classes, which was not as apparent in previous studies using only culture-based methods that target a limited number of bacterial species but see [10]. As gastrointestinal bacteria have important functions in digestion, immune functions and general health, the characterisation of the acquisition of bacteria comprises a crucial component in achieving a more comprehensive understanding of the causes and consequences of variation in bacterial communities in wild animals.

\section{Additional files}

Additional file 1: Identity of OTUs isolated from control samples that are assumed to be contaminants.

Additional file 2: 235 rRNA phylogenetic trees for the 76 identified bacterial OTUs isolated from black-legged kittiwake cloacae.

\section{Abbreviations}

ARISA: Automated ribosomal intergenic spacer analysis; BLAST: Basic local alignment search tool; DNA: Deoxyribonucleic acid; dNTPS: Deoxynucleotide triphosphates; IGS: Intergenic spacer; $\mathrm{MgCl}_{2}$ : Magnesium chloride; OTU: Operational taxonomic unit; PCR: Polymerase chain reaction; rRNA: ribosomal ribonucleic acid; SD: Standard deviation.

\section{Competing interests}

The authors declare that they have no competing interests.

\section{Authors' contributions}

The study was designed by JW, RW and ED and fieldwork was performed by JW, TM, SL, PB and SH. HB conducted the laboratory work. YM provided advice for the genetic analyses and WvD and HB analysed the data. WVD 
wrote the manuscript. All authors contributed to and approved the final manuscript.

\section{Acknowledgements}

We thank François Bailly for field assistance, Florian Sammer for assistance with molecular work, Anne Hloch for assistance creating the figures and Andrew Ramey for comments on an earlier version of this manuscript. Experiments were carried out in accordance with United States' laws and under permits from the U.S. Fish and Wildlife Service and State of Alaska. Field data collection was financed by the French Polar Institute Paul-Emile Victor (IPEV, program 1162). This laboratory component of this study was supported by the University of Veterinary Medicine Vienna and the Austrian Science Foundation (FWF) Project 20401 to RHW. The lab EDB, which hosts five of the co-authors (JW, TM, SL, PB and ED) is part of the "Laboratoire d'Excellence (LABEX)" entitled TULIP (ANR -10-LABX-41). Any use of trade names is for descriptive purposes only and does not imply endorsement of the U.S. Government.

\section{Author details}

${ }^{1}$ Konrad Lorenz Institute of Ethology, Department of Integrative Biology and Evolution, University of Veterinary Medicine Vienna, Savoyenstrasse 1a, 1160 Vienna, Austria. ${ }^{2}$ CNRS-UPS-ENFA; Laboratoire Évolution \& Diversité Biologique (EDB), UMR 5174, 118 Route de Narbonne, F-31062 Toulouse, France. ${ }^{3}$ US Geological Survey, Alaska Science Center, 4210 University Drive, Anchorage, AK 99508 USA.

Received: 16 November 2012 Accepted: 14 March 2013 Published: 25 March 2013

\section{References}

1. Archie EA, Theis TR: Animal behaviour meets microbial ecology. Anim Behav 2011, 82:425-436.

2. Hill MJ: Intestinal flora and endogenous vitamin synthesis. Europ J Cancer Prevent, Supp 1997, 6:S43-S45.

3. Ley RE, Lozupone CA, Hamady M, Knight R, Gordon Jl: Worlds within worlds: evolution of the vertebrate gut microbiota. Nat Rev Microbiol 2008, 6:776-788.

4. Macpherson AJ, Harris NL: Interactions between commensal intestinal bacteria and the immune system. Nat Rev Immun 2004, 4:478-485.

5. Benskin $\mathrm{CMH}$, Wilson K, Jones K, Hartley IR: Bacterial pathogens in wild birds: a review of the frequency and effects of infection. Biol Rev Camb Philos Soc 2009, 84:349-373.

6. Ezenwa VO, Gerardo NM, Inouye DW, Medina M, Xavier JB: Animal behavior and the microbiome. Science 2012, 338:198-199.

7. Kohl KD: Diversity and function of the avian gut microbiota. J Comp Physiol B - Biochem Syst Environ Physiol 2012, 182:591-602.

8. Macfarlane GT, Macfarlane LE: Acquisition, evolution and maintenance of the normal gut microbiota. Dig Dis 2009, 27:90-98.

9. Palmer C, Bik EM, DiGiulio DB, Relman DA, Brown PO: Development of the human infant intestinal microbiota. PLOS Biol 2007, 5:1556-1573.

10. Godoy-Vitorino F, Goldfarb KC, Brodie EL, Garcia-Amado MA, Michelangeli F, Dominguez-Bello MG: Developmental microbial ecology of the crop of the folivorous hoatzin. Isme J 2010, 4:611-620.

11. Sharon G, Segal D, Ringo JM, Hefetz A, Zilber-Rosenberg I, Rosenberg E: Commensal bacteria play a role in mating preference of Drosophila melanogaster. Proc Natl Acad Sci USA 2010, 107:20051-20056.

12. Stecher B, Hardt WD: Mechanisms controlling pathogen colonization of the gut. Curr Opin Microbiol 2011, 14:82-91.

13. White J, Mirleau P, Danchin E, Mulard H, Hatch SA, Heeb P, Wagner RH: Sexually transmitted bacteria affect female cloacal assemblages in a wild bird. Ecol Lett 2010, 13:1515.

14. Lombardo MP: On the evolution of sexually transmitted diseases in birds. J Avian Biol 1998, 29:314-321.

15. Sheldon BC: Sexually transmitted disease in birds: occurrence and evolutionary significance. Philos Trans Biol Sci 1993, 339:491-497.

16. Lombardo MP, Thorpe PA, Power HW: The beneficial sexually transmitted microbe hypothesis of avian copulation. Behav Ecol 1999, 10:333-337.

17. Gong J, Yu H, Liu T, Gill JJ, Chambers JR, Wheatcroft R, Sabour PM: Effects of zinc bacitracin, bird age and access to range on bacterial microbiota in the ileum and caeca of broiler chickens. J Appl Microbio/ 2008, 104:1372-1382.
18. Lan Y, Verstegen MWA, Tamminga S, Williams BA: The role of the commensal gut microbial community in broiler chickens. World Poultry Sci J 2005, 61:95-104.

19. Scupham AJ: Succession in the intestinal microbiota of preadolescent turkeys. FEMS Microbiol Ecol 2007, 60:136-147.

20. Tanikawa T, Shoji N, Sonohara N, Saito S, Shimura Y, Fukushima J, Inamoto $\mathrm{T}$ : Aging transition of the bacterial community structure in the chick ceca. Poult Sci 2011, 90:1004-1008.

21. Villers LM, Jang SS, Lent CL, Lewin-Koh SC, Norosoarinaivo JA: Survey and comparison of major intestinal flora in captive and wild ring-tailed lemur (Lemur catta) populations. Am J Primat 2008, 70:175-184.

22. Xenoulis PG, Gray PL, Brightsmith D, Palculict B, Hoppes S, Steiner JM, Tizard I, Suchodolski JS: Molecular characterization of the cloacal microbiota of wild and captive parrots. Vet Microbiol 2010, 146:320-325.

23. Gonzalez-Braojos S, Vela Al, Ruiz-de-Castaneda R, Briones V, Moreno J: Agerelated changes in abundance of enterococci and Enterobacteriaceae in Pied Flycatcher (Ficedula hypoleuca) nestlings and their association with growth. J Ornithol 2012, 153:181-188.

24. Janiga M, Sedlarova A, Rigg R, Novotna M: Patterns of prevalence among bacterial communities of alpine accentors (Prunella collaris) in the Tatra Mountains. J Ornithol 2007, 148:135-143.

25. Lombardo MP, Thorpe PA, Cichewicz R, Henshaw M, Millard C, Steen C, Zeller TK: Communities of cloacal bacteria in Tree Swallow families. Condor 1996, 98:167-172.

26. Mills TK, Lombardo MP, Thorpe PA: Microbial colonization of the cloacae of nestling tree swallows. Auk 1999, 116:947-956.

27. Meade GC: Bacteria in the gastrointestinal tract of birds. In Gastrointestinal microbiology. Edited by Mackie RI, White BA, Isaacson RE. New York: Chapman and Hall; 1997.

28. Ranjard L, Brothier E, Nazaret S: Sequencing bands of ribosomal intergenic spacer analysis fingerprints for characterization and microscale distribution of soil bacterium populations responding to mercury spiking. Appl Environ Microbiol 2000, 66:5334-5339.

29. Kovacs A, Yacoby K, Gophna U: A systematic assessment of automated ribosomal intergenic spacer analysis (ARISA) as a tool for estimating bacterial richness. Res Microbiol 2010, 161:192-197.

30. Altschul SF, Gish W, Miller W, Myers EW, Lipman DJ: Basic local alignment search tool. J Mol Biol 1990, 215:403-410.

31. Jobb G, von Haeseler A, Strimmer K: TREEFINDER: A powerful graphical analysis environment for molecular phylogenetics. BMC Evol Biol 2004, 4:18.

32. Huelsenbeck JP, Ronquist F: MRBAYES: Bayesian inference of phylogeny. Bioinformatics 2001, 17:754-755.

33. Lozupone C, Hamady M, Knight R: UniFrac - an online tool for comparing microbial community diversity in a phylogenetic context. $B M C$ Bioinformatics 2006, 7:371.

34. Banning NC, Gleeson DB, Grigg AH, Grant CD, Andersen GL, Brodie EL, Murphy DV: Soil microbial community successional patterns during forest ecosystem restoration. Appl Environ Microbiol 2011, 77:6158-6164.

35. Corrigan A, Horgan K, Clipson N, Murphy RA: Effect of dietary supplementation with a Saccharomyces cerevisiae mannan oligosaccharide on the bacterial community structure of broiler cecal contents. Appl Environ Microbiol 2011, 77:6653-6662.

36. Schottner S, Pfitzner B, Grunke S, Rasheed M, Wild C, Ramette A: Drivers of bacterial diversity dynamics in permeable carbonate and silicate coral reef sands from the Red Sea. Environ Microbiol 2011, 13:1815-1826.

37. Weimer PJ, Stevenson DM, Mantovani HC, Man SLC: Host specificity of the ruminal bacterial community in the dairy cow following near-total exchange of ruminal contents. J Dairy Sci 2011, 93:5902-5912.

38. White J, Richard M, Massot M, Meylan S: Cloacal bacterial diversity increases with multiple mates: evidence of sexual transmission in female common lizards. PLOS ONE 2011, 6:e22339.

39. Chandler DP, Fredrickson JK, Brockman FJ: Effect of PCR template concentration on the composition and distribution of total community 16S rDNA clone libraries. Mol Ecol 1997, 6:475-482.

40. Scupham AJ, Jones JA, Wesley IV: Comparison of DNA extraction methods for analysis of turkey cecal microbiota. J Appl Microbiol 2007, 102:401-409.

41. Haussmann MF, Winkler DW, Huntington CE, Vleck D, Sanneman CE, Hanley D, Vleck CM: Cell-mediated immunosenescence in birds. Oecologia 2005, 145:270-275.

42. Lavoie ET, Sorrell EM, Perez DR, Ottinger MA: Immunosenescence and age-related susceptibility to influenza virus in Japanese quail. Dev Comp Immunol 2007, 31:407-414. 
43. Noreen E, Bourgeon S, Bech C: Growing old with the immune system: a study of immunosenescence in the zebra finch (Taeniopygia guttata). J Comp Physiol B - Biochem Syst Environ Physiol 2011, 181:649-656.

44. Flint JF, Garner MR: Feeding beneficial bacteria: A natural solution for increasing efficiency and decreasing pathogens in animal agriculture. J Appl Poultry Res 2009, 18:367-378.

45. Koenen ME, Kramer J, van der Hulst R, Heres L, Jeurissen SH, Boersma WJ: Immunomodulation by probiotic lactobacilli in layer- and meat-type chickens. Brit Poultry Sci 2004, 45:355-366.

46. Duriez P, Clermont O, Bonacorsi S, Bingen E, Chaventre A, Elion J, Picard B, Denamur E: Commensal Escherichia coli isolates are phylogenetically distributed among geographically distinct human populations. Microbiology-Sgm 2001, 147:1671-1676.

47. Blanco G, Lemus JA, Grande J: Faecal bacteria associated with different diets of wintering red kites: influence of livestock carcass dumps in microflora alteration and pathogen acquisition. J Appl Ecol 2006, 43:990-998.

48. Klomp JE, Murphy MT, Smith SB, McKay JE, Ferrera I, Reysenbach AL: Cloacal microbial communities of female spotted towhees Pipilo maculatus: microgeographic variation and individual sources of variability. J Avian Biol 2008, 39:530-538.

49. Banks JC, Cary SC, Hogg ID: The phylogeography of Adelie penguin faecal flora. Environ Microbiol 2009, 11:577-588.

50. Ruiz-de-Castaneda R, Vela Al, Lobato E, Briones V, Moreno J: Prevalence of potentially pathogenic culturable bacteria on eggshells and in cloacae of female Pied Flycatchers in a temperate habitat in central Spain. J Field Ornithol 2011, 82:215-224.

51. Arumugam M, Raes J, Pelletier E, Le Paslier D, Yamada T, Mende DR, Fernandes GR, Tap J, Bruls T, Batto JM, et al: Enterotypes of the human gut microbiome. Nature 2011, 473:174-180.

doi:10.1186/1472-6785-13-11

Cite this article as: van Dongen et al: Age-related differences in the

cloacal microbiota of a wild bird species. BMC Ecology 2013 13:11.

\section{Submit your next manuscript to BioMed Central and take full advantage of:}

- Convenient online submission

- Thorough peer review

- No space constraints or color figure charges

- Immediate publication on acceptance

- Inclusion in PubMed, CAS, Scopus and Google Scholar

- Research which is freely available for redistribution 